\title{
RESEARCH
}

Open Access

\section{Pathological staging of chorioamnionitis contributes to complications in preterm infants}

Jiayu Miao ${ }^{1 \dagger}$, Zhuxiao Ren ${ }^{1 \dagger}$, Yunbei Rao ${ }^{2}$, Xin Xia ${ }^{1}$, Jianlan Wang ${ }^{1}$, Fang $X^{1}{ }^{1}$, Xiaoling Zhang ${ }^{1}$ and Jie Yang ${ }^{{ }^{*}}$

\begin{abstract}
Objective: To investigate the effect of pathological staging of chorioamnionitis (CA) on complications in preterm infants;

Methods: A single-center, retrospective study was conducted to choose singleton preterm infants (gestational age < 37 weeks) from the Department of Obstetrics and Gynecology in our hospital from December 2016 to December 2017. The basic data and placental pathological results were retrospectively collected. According to the placental pathological results of whether inflammation infiltrating amnion, CA 0/I phase was classified into nonamnionitis group, CA II/III phase was classified into amnionitis group, the incidence of common complications in preterm infants was compared. Further, logistic regression was used to analyze the effects of amnionitis on complications after being adjusted to gestational age, birth weight and thrombocytopenia.
\end{abstract}

Results: A total of 221 preterm infants were enrolled, including 186 cases in non-amnionitis group and 35 cases in amnionitis group. The gestational age of amnionitis group (32.00 \pm 2.71 weeks) was significantly lower than nonamnionitis group $(34.14 \pm 2.06$ weeks), birth weight $(1.93 \pm 0.64 \mathrm{~kg})$ was significantly lower than that of nonamnionitis group $(2.26 \pm 0.58 \mathrm{~kg})$, and the hospital stay in amnionitis group was significantly longer $(25.71 \pm 19.23$ days), all of the difference above was statistically significant $(P<0.05)$. The incidence of intraventricular hemorrhage $(\mathrm{IVH})$ in amnionitis group (37.14\%) was significantly higher than that in non-amnionitis group (13.98\%) $(P=0.002)$, and the risk of IVH was significantly increased by amnionitis ( $\mathrm{OR}=3.636,95 \% \mathrm{Cl}: 1.632-8.102)$; after correction of gestational age, birth weight and thrombocytopenia, the risk of IVH was still significantly increased $(\mathrm{OR}=2.471, P=$ 0.046, 95\% Cl: 1.015-6.015). And the late-onset IVH was more common ( $P=0.009)$.

Conclusion: Amnionitis leads to a significant reduction in gestational age and birth weight in preterm infants, and it is an independent risk factor for IVH.

Keywords: Chorioamnionitis, Amnionitis, Complications, Preterm infants, Intraventricular hemorrhage

\section{Background}

Chorioamnionitis, an inflammatory status of the intrauterine or fetal membrane, were divided into histological chorioamnionitis and clinical chorioamnionitis [1]. It is

\footnotetext{
* Correspondence: jieyang0830@yeah.net

${ }^{\dagger}$ Jiayu Miao and Zhuxiao Ren contributed equally to this work.

'Department of Neonatology, Guangdong Women and Children Hospital, Guangzhou, China

Full list of author information is available at the end of the article
}

well known that chorioamnionitis can increase the incidence of intraventricular hemorrhage (IVH), periventricular leukomalacia (PVL), bronchopulmonary dysplasia (BPD), retinopathy (ROP), early-onset sepsis (EOS), late-onset sepsis (LOS), and necrotizing entercolitis (NEC) [2]. Chorioamnionitis leads to an inflammatory response in the placenta, inflammatory cells and factors could induce vascular endothelial injury leading 
to perfusion defect in placenta [3]. Placental malperfusion was associated with earlier preterm birth accompanied by impaired fetal growth and severe neonatal complications [4]. On the other hand, Chorioamnionitis can cause fetal inflammatory response syndrome (FIRS), which is a subclinical state caused by the activation of the fetal immune system and the release of a large number of inflammatory factors (IL-6, IL-1, IL-8, TNF- $\alpha$ ). These inflammatory factors could interfere with the normal expression of fetal brain cytokines, leading to brain damage in preterm infants [5]. Some preclinical studies showed that fetal lung epithelial damage caused by chorioamnionitis could lead to impaired vascular osmotic pressure and apoptosis [6], which increased the secretion of lung surface proteins, but could also make the alveolar cell wall thinner $[7,8]$. Similarly, inflammatory factors could damage the fetal lung capillary endothelial cells, resulting in damage to the alveoli, vascular structure and stagnation of alveolarization, which ultimately leads to the occurrence of BPD $[9,10]$. Gantert. et al. confirmed that premature delivery and intrauterine inflammation affected the maturation of the fetal intestinal barrier, the intestinal tight junction protein-1 (TJP-1) damaged by bacterial toxins during gestation, resulting in an easy access of microbial toxins to the mucosa and the inner layers of the gut ante- and postnatally [11].

In summary, Chorioamnionitis is associated with adverse outcomes in preterm birth. Although, the effect of chorioamnionitis on complications in preterm infants has been widely studied, the pathological staging on them has been rarely published. Therefore, this study aimed to investigate the effect of pathological staging of chorioamnionitis on complications in preterm infants.

\section{Methods}

\section{Study population}

A single-center, retrospective study was conducted. Singleton infants (gestational age $<37$ weeks) who were given birth in our hospital from December 2016 to December 2017 were selected as study population, cases of genetic metabolic diseases, nervous system malformations, and stillbirth were eliminated. The inclusion criteria for sending placenta for pathology investigation in our center are one of the following situations: 1 . Mother with gestational diabetes or preeclampsia; 2 . Mother had fever a week before or during delivery; 3. Macroscopic abnormity during delivery. In addition, informed consent for pathology analysis should be obtained. The protocol was approved by Ethics Committee of Guangdong Women and Children's Hospital (201801053).

\section{Histopathological examination}

After delivery, the whole placenta, fetal membrane and umbilical cord were fixed in $4 \%$ formaldehyde for $48 \mathrm{~h}$, the quality of the placenta, the length and the attachment of umbilical cord, the number of blood vessels were evaluated, and the placenta was cut at intervals of $1 \mathrm{~cm}$. All specimens were embedded in paraffin, sectioned, and routine Hematoxylin-Eosin Staining (HE staining).

The criteria for histological chorioamnionitis: Stage 0 (CA0): Normal placenta; Stage 1 (CAI): acute inflammation of the chorioamniotic membranes, in which neutrophilic infiltration is limited to the chorion; Stage 2 (CAII): acute inflammation of the chorioamniotic membranes, showing neutrophilic migration into the amniotic connective tissue (asterisk); Stage 3 (CAIII): acute inflammation of the chorioamniotic membranes, whose characteristic is the amnion epithelial necrosis (arrows) [12].

According to the placental pathological results of whether inflammation infiltrating amnion, CA 0/I phase was classified into non-amnionitis group, CA II/III phase was classified into amnionitis group.

\section{Clinical characteristics}

The gestational age (gestational age in our data was based on date of last menstrual period with confirmation by ultrasound), Apgar score, birth weight, blood routine, C-reactive protein, procalcitonin and blood culture results of each premature infant were collected, and the disease of each system during hospitalization was observed. The relevant complications were as follows [13]:

Intraventricular hemorrhage (IVH), periventricular leukomalacia (PVL) are common complications in preterm infants, which can be detected by serial head ultrasound and MRI, the first is recommended between 5 and 8 days, 21 and 28 days, between 34 and 36 weeks of corrected gestational age [14]. Those with IVH born $\leq 3$ $\mathrm{d}$ were defined as early-onset intraventricular hemorrhage, and $>3 \mathrm{~d}$ were defined as late-onset intraventricular hemorrhage [15].

The sepsis in this study requires positive clinical or laboratory screen and positive culture. Early-onset sepsis (EOS) occurred during the first $72 \mathrm{~h}$ of life, Late-onset sepsis (LOS) refers to sepsis that occurred between day of 4 and 120 [16].

Bronchopulmonary dysplasia (BPD) is defined as oxygen dependency at 36 weeks of corrected gestational age, the main causes including damage of alveolar, vascular structural and stagnation of alveolarization.

NEC is a syndrome manifested as abdominal distension, vomiting, diarrhea and Bloody stools, severe cases even develop shock and multiple systemic organ failure, can be diagnosed by abdominal piece, usually characterized by gas accumulation in the intestinal wall.

Retinopathy of prematurity (ROP) refers to retinal vasoconstriction, obstruction and retinal vascular 
hypoxia, leading to a large number of angiogenic factors, stimulating neovascularization form.

Neonatal thrombocytopenia: The platelet count $\leq 150^{*} 10^{9} / \mathrm{L}$ in all healthy newborn infants, regardless of gestational age [17].

\section{Statistical analysis}

One Sample Kolmogorov-Smirnov test was performed for normality of distributions, and the normal distribution data was represented as the mean \pm standard deviation (SD). Dichotomous data was expressed by the frequency and relevant percentage. Maternal and neonates' characteristics were compared using $x^{2}$ and $t$ tests as appropriate. Variables with statistical significance after univariate analysis and factors which could affect neonatal complications were further adjusted by multivariate logistic regression, the effect size is expressed in OR and $95 \%$ CI. Significance was accepted at $p<0.05$. All analyses were performed by using SPSS version 19.0 software.

\section{Results}

\section{General situation}

A total of 221 maternal placenta were detected in this study. Including 186 cases in non-amnionitis group, 35 cases in amnionitis group. The gestational age of amnionitis group ( $32.00 \pm 2.71$ weeks) was significantly lower than non-amnionitis group (34.14 \pm 2.06 weeks), birth weight $(1.93 \pm 0.64 \mathrm{~kg})$ was significantly lower than that of non-amnionitis group $(2.26 \pm 0.58 \mathrm{~kg})$, and the hospital stay in amnionitis group (25.71 \pm 19.23 days) was significantly longer than that in non-amnionitis group (18.84 \pm 16.80 days), the difference was statistically significant $(P<0.05)$. However, there were no significant difference in maternal age, placental weight, use of antenatal glucocorticoids, premature rupture of membranes, mode of delivery, gender, thrombocytopenia, and Apgar scores $(P>0.05)$. (As shown as in Table 1$)$.

\section{Neonatal outcome}

Among the 221 patients, the incidence of intraventricular hemorrhage (IVH) in amnionitis group (37.14\%) was significantly higher than that in non-amnionitis group $(13.98 \%)$, the difference was statistically significant $(\mathrm{OR}=$ 3.636, $P=0.002$, 95\%CI: 1.632-8.102); After correction of gestational age, birth weight and thrombocytopeniathe three factor that are regarded to affect the IVH incidence, the risk of IVH was still significantly increased $(\mathrm{OR}=2.471, P=0.046,95 \% \mathrm{CI}: 1.015-6.015)$, and it more likely to cause late-onset IVH $(P=0.009)$ (as shown as in Table 2); However, there was no difference between the two groups on the incidence of necrotizing enterocolitis (NEC), bronchopulmonary dysplasia (BPD), retinopathy of prematurity (ROP), sepsis $(P>0.05)$ (as shown as in Table 3). Therefore, it is indicated that histological amnionitis was an independent risk factor for intraventricular hemorrhage in premature infants, which is not affected by gestational age, birth weight and thrombocytopenia, and it had no significant effects on other common complications related to premature birth $(P<0.05)$.

\section{Discussion}

\section{Principal findings}

Intra-amniotic infection has been considered to be the cause of acute histologic chorioamnionitis and funisitis, and funisitis is the hallmarks for the FIRS, which is associated with the preterm labor, a higher rate of neonatal morbidity (after adjustment for gestational age), and multi-organ fetal involvement $[12,18]$. In this

Table 1 The clinical characteristics of non- amnionitis group and amnionitis group

\begin{tabular}{llll}
\hline Clinical characteristics & Non-amnionitis group (186) & Amnionitis group (35) & P \\
\hline Maternal age(y) & $30.62 \pm 5.38$ & $30.89 \pm 6.80$ & 0.797 \\
Gestational age(d) & $34.14 \pm 2.06$ & $32.00 \pm 2.71$ & $<0.001$ \\
Placental weight(g) & $480.71 \pm 150.43$ & $475.86 \pm 106.68$ & 0.856 \\
Birth weight (kg) & $2.26 \pm 0.58$ & $1.93 \pm 0.64$ & 0.003 \\
Antenatal glucocorticoids,n,(\%) & $46(24.7 \%)$ & $12(34.3 \%)$ & 0.239 \\
Premature rupture,n,(\%) & $64(34.0 \%)$ & $16(45.7 \%)$ & 0.186 \\
Cesarean section, $\mathrm{n},(\%)$ & $96(51.6 \%)$ & $16(45.7 \%)$ & 0.522 \\
Male, $\mathrm{n},(\%)$ & $107(57.5 \%)$ & $16(45.7 \%)$ & 0.197 \\
Apgar score 1 min $\leq 7, \mathrm{n},(\%)$ & $7(3.8 \%)$ & $3(8.6 \%)$ & 0.417 \\
Apgar score 5 min $\leq 7, \mathrm{n},(\%)$ & $4(2.2 \%)$ & $1(2.9 \%)$ & 1.000 \\
PLT, $\leq 150 \times 10^{9} / \mathrm{L}, \mathrm{n},(\%)$ & $12(6.5 \%)$ & $1(2.9 \%)$ & 0.662 \\
Hospitalization(d) & $18.84 \pm 16.80$ & $25.71 \pm 19.23$ & 0.031 \\
\hline
\end{tabular}

PLT Platelet. Data are expressed as $\mathrm{n}(\%)$ or mean \pm standard deviation. The measurement data were analyzed by independent sample $\mathrm{t}$ test, and the count data were analyzed by chi-square test. ${ }^{*} P<0.05$, the difference was statistically significant 
Table 2 Comparison of chorioamnionitis staging and IVH staging

\begin{tabular}{llll}
\hline IVH staging & Non-amnionitis group (186) & Amnionitis group (35) & P \\
\hline $\mathrm{IVH}, \mathrm{n},(\%)$ & $26(13.98 \%)$ & $13(37.14 \%)$ & 0.002 \\
Early-onset, $\mathrm{n},(\%)$ & $14(7.53 \%)$ & $6(17.14 \%)$ & 0.069 \\
Late-onset, $\mathrm{n},(\%)$ & $12(6.45 \%)$ & $7(20.00 \%)$ & 0.009 \\
\hline
\end{tabular}

IVH Intraventricular hemorrhage. Data are expressed as $\mathrm{n}(\%)$.The count data were analyzed by chi-square test. ${ }^{*} P<0.05$, the difference was statistically significant

prospective study performed on 221 preterm newborns, we assessed their birth outcomes and common complications following amnionitis exposure. The key findings of this study are the following: (1) Placental amnionitis could lead to intrauterine growth retardation, associated with lower birth weight and preterm birth. (2) Preterms in amnionitis group had a higher occurrence of IVH, after correction of the common risk factors in IVH, including gestational age, birth weight and thrombocytopenia, amnionitis is still an independent risk factor for IVH.

\section{Strengths and weaknesses}

The study has three strengths. First, we divided the studying population into non-amnionitis and amnionitis groups, the classification was not reported previously and it could highlight the effect of severe intrauterine inflammation on birth outcomes and complications of preterm compared with other previous studies. The placental tissue inflammation continues to progress and the intensity of the inflammation increases, which can cause severe amniotic inflammation and fetal inflammatory response syndrome, it is believed that the latter is closely related to the occurrence of premature delivery and fetal complications $[19,20]$. Second, gestational age was confirmed by ultrasound, which is more rigorous. Third, histological chorioamnionitis may still be present in case of premature labor, even in the absence of clinical signs and premature rupture of membranes. Therefore, we use histological criteria for amnionitis. However, this study also has some limitations. First, the number of amniotitis cases was limited and no placental pathology was done in stillbirth. Second, except for postpartum factors, some prenatal factors may also cause IVH, such as placental vascular disease, hemodynamics, which our study did not evaluate. Third, Gomez et al. found that the content of IL-6 in fetal umbilical cord blood was an independent risk factor for severe complications in FIRS, IL- $6>11 \mathrm{pg} / \mathrm{mL}$ as FIRS reference standard [21], but in our study, we didn't give the evidence between CA and FIRS. Therefore, we may conduct a larger study to assess the effect of pathological staging of CA on preterm infants.

\section{Comparison with previous studies}

The placenta functions are important for development of fetal multiple organs and systems. Placental dysfunction would affect the fetus, resulting in fetal growth restriction, premature and neurodevelopmental abnormalities [22, 23]. A series of studies have incorporated placental features into studies of stillbirth, preterm delivery and growth restriction [24-27]. Erdemir et al. revealed that histological chorioamnionitis led to earlier gestational age and lower birth weight [28]. Zanardo V et al. reported that the incidence of IVH in premature infants born to pregnant women with CA could reach $16.1 \%$, and the stage 3 of placental inflammation could increase the risk of IVH by 3.2 times [29]. This study found that the CAII/III is a risk factor for IVH in premature infants, which is consistent with reports in the literature. However, for the staging of IVH, many studies have not specifically emphasized the role of chorioamnionitis [30-32], IVH of early-onset is known to be associated with greater neonatal mortality rate and the risk of subsequent neurodevelopmental disability in survivors $[30,33]$. Therefore, it is important to understand

Table 3 Neonatal outcomes of non- amnionitis group and amnionitis group

\begin{tabular}{|c|c|c|c|c|c|c|}
\hline Outcomes & Non- amnionitis group (186) & Amnionitis group (35) & $P$ & OR(95\%Cl) & ${ }_{P}^{*}$ Adjusted & ${ }^{*}$ Adjusted $\mathrm{OR}(95 \% \mathrm{Cl})$ \\
\hline IVH,n, (\%) & 26(13.98\%) & $13(37.14 \%)$ & 0.002 & $3.636(1.632-8.102)$ & 0.046 & $2.471(1.015-6.015)$ \\
\hline BPD,n,(\%) & $7(3.8 \%)$ & $3(8.6 \%)$ & 0.222 & $2.397(0.589-9.759)$ & 0.444 & $0.493(0.080-3.015)$ \\
\hline NEC,n, (\%) & $20(10.8 \%)$ & $3(8.6 \%)$ & 0.699 & $0.778(0.218-2.774)$ & 0.117 & $0.322(0.078-1.331)$ \\
\hline $\mathrm{ROP}, \mathrm{n},(\%)$ & 16(8.6\%) & $2(5.7 \%)$ & 0.569 & $0.644(0.141-2.934)$ & 0.104 & $0.251(0.048-1.328)$ \\
\hline Sepsis, n, (\%) & $1(0.5 \%)$ & $1(2.9 \%)$ & 0.235 & $5.441(0.332-89.106)$ & 0.415 & $3.740(0.157-89.228)$ \\
\hline
\end{tabular}

IVH Intraventricular hemorrhage, BPD Bronchopulmonary dysplasia, NEC Necrotizing entercolitis, ROP Retinopathy. Data are expressed as $\mathrm{n}$ (\%).The count data were analyzed by chi-square test. ${ }^{*} P<0.05$, the difference was statistically significant. ${ }^{*}$ Adjusted OR (adjusted for GA and BW, for IVH adjusted for GA and BW and thrombocytopenia) 
whether CA plays a role in the pathogenesis of earlyonset IVH as opposed to late-onset IVH. Subrata et al. showed that chorioamnionitis was not associated with increased risk of early IVH [15]; Lou reported that the higher incidence of IVH associated with CA might have been mostly of late onset [34]. In our study, the lateonset IVH was more predominant $(P<0.05)$, which was the same with Lou. Chorioamnionitis leads to the release of vasoactive inflammatory cytokines which can mediate blood-brain barrier alterations, intra-vascular cells adhesion, coagulation, and thrombosis, conversely, this can lead to endothelial damage. If the severity of systemic and local hemodynamic changes increased in infants accompanied by cerebral endothelial damage, the enhanced late neonatal inflammatory response syndrome leads to the development of late onset IVH [15].

Earlier studies have focused mainly on the respiratory and neurological outcomes, chorioamnionitis have more recently been described in several other organs and systems. Watterberg et al. were the first to show that exposure to placental inflammation decreased the risk of RDS, but a subsequent increase in chronic lung injury (BPD) [35]. But a few studies have reported no relationship between placental inflammation and RDS [36], but our research lacks of the investigation on RDS. Some studies indicate that histologic chorioamnionitis could increase the risk of early onset sepsis and reduce the risk of late onset sepsis in preterm infants [37, 38], However, we have not been able to explore the early-onset and late-onset in our study. In addition, there were studies reported that chorioamnionitis seemed to contribute importantly to the development of ROP and NEC [39, 40], which our study still has not been able to get same findings.

Antenatal factors are also important to chorioamnionitis and neonatal morbidity. Antenatal steroids were reported to reduce negative effects of CA, such as IVH and RDS, as well as mortality by suppression of inflammation [41]. In this study, antenatal steroids did not change the frequency of CA in pregnant women, but it could be evaluated for neonatal morbidities by multiple regression analysis. Erdemir and his colleague analyzed the effect of many maternal risk factors on CA, neonatal morbidity and mortality rates, not only common complications, but also the severity of illness such as ventilation duration, surfactant therapy [28], which is lacking in our study.

\section{Meaning of the study}

This study revealed the adverse birth outcomes caused by severe intrauterine inflammation exposure, which could help to highlight the importance of placental pathology examinations, therefore guiding clinical management and reducing the devastating burden of adverse birth outcomes.

\section{Unanswered questions and future research}

In this study, there is a lack of the underlying mechanisms of stage of chorioamnionitis on neonatal adverse outcomes. For example, how the chemotactic signals in the amniotic cavity induced the amniotic inflammation, the relationship of FIRS and IVH. Therefore, additional preclinical studies are needed to explore these problems, and the number of included amnionitis cases needs to be enlarged.

\section{Conclusions}

Histological chorioamnionitis is closed to various complications of premature infants, especially intraventricular hemorrhage IVH associated with a fetal inflammatory response syndrome [42]. Clinically, the effect of intrauterine inflammation on premature infants should be emphasized. For mothers with chorioamnionitis, focus on monitoring, especially that the placenta should be routine pathological examination, improve the detection rate of chorioamnionitis, reduce the sequelae of nervous system in premature infants.

\section{Acknowledgements}

Not applicable.

\section{Authors' contributions}

Jiayu Miao collected all data and wrote the manuscript, Zhuxiao Ren and Yunbei Rao help analyze the data, Xin Xia, Jianlan Wang, Fang Xu and Xiaoling Zhang collected the references and revised the manuscript, Jie Yang revised and reported the manuscript. All authors read and approved the final manuscript.

\section{Funding}

This work was supported by the Guang Zhou Science and Technology Grant under Grant [No. 201707010398].

Availability of data and materials

Please contact author for data requests.

Ethics approval and consent to participate

The protocol was approved by Ethics Committee of Guangdong Women and Children's Hospital(201801053).

Consent for publication

Not applicable.

Competing interests

The authors declare that they have no competing interests.

Author details

'Department of Neonatology, Guangdong Women and Children Hospital, Guangzhou, China. ²Department of Neonatology, Guangzhou Medical University Second Affiliated Hospital, Guangzhou, China.

Received: 21 January 2020 Accepted: 2 September 2020

Published online: 11 September 2020

\section{References}

1. Hagberg H, Wennerholm UB, et al. Sequelae of chorioamnionitis. Curr Opin Infect Dis. 2002;15:301-6. 
2. Thomas W, Speer CP. Chorioamnionitis:important risk factor or innocent bystander for neonatal outcome? Neonatology. 2011;99:177-187.

3. Kaukola T. Herva, et al. population cohort associating Chorioamnionitis, cord inflammatory cytokines and neurologic outcome in very preterm, extremely low birth weight infants. Pediatr Res. 2006;59(3):478-83.

4. Catov $\mathrm{JM}$, Scifres $\mathrm{CM}$, et al. Neonatal outcomes following preterm birth classified according to placental features. Am J Obstet Gynecol. 2017;216(4): 411.e1-411.e14.

5. Meyer U, Feldon J, Yee BK. A review of the fetal brain cytokine imbalance hypothesis of schizophrenia. Schizophr Bull. 2009;35(5):959-72.

6. Kramer BW, Kramer S, Ikegami M, et al. Injury, inflammation, and remodeling in fetal sheep lung after intra-amniotic endotoxin. Am J Physiol Lung Cell Mol Physiol. 2002;283:L452-9. https://doi.org/10.1152/ajplung.00407.2001.

7. Kallapur SG, Willet KE, Jobe AH, et al. Intra-amniotic endotoxin: chorioamnionitis precedes lung maturation in preterm lambs. Am J Physiol Lung Cell Mol Physiol. 2001;280:L527-36.

8. Willet KE, Jobe AH, Ikegami M, et al. Antenatal endotoxin and glucocorticoid effects on lung morphometry in preterm lambs. Pediatr Res. 2000;48:782-8. https://doi.org/10.1203/00006450-200012000-00013.

9. Khankin EV, Mandala M, Colton I, et al. Hemodynamic, vascular, and reproductive impact of FMS-like tyrosine kinase 1 (FLT1) blockade onthe uteroplacental circulation during normal mouse pregnancy. []]. Biol Reprod. 2012;86(2):57.

10. Weissgerber TL, Rajakumar A, Myerski AC, et al. Vascular Pool of releasable soluble VEGF Receptor-1 (sFlt1) in women with previous preeclampsia and uncomplicated pregnancy. J Clin Endocrinol Metabol. 2014;99(3):978-87.

11. Gantert M, Been JV, Gavilanes AW, et al. Chorioamnionitis: a multiorgan disease of the fetus? J Perinatol. 2010;30:S21-30.

12. Kim CJ, Romero R, Chaemsaithong P, et al. Acute chorioamnionitis and funisitis: definition, pathologic features, and clinical significance. Am J Obstetr Gynecol. 2015;213(4):S29-52.

13. Xiaomei S, Maohong Y, Xiaoshan Q. Practical neonology. Beijing: People 's Medical Publishing House; 2010. p. 222, 395, 4477, 534, 715. (In Chinese).

14. Schmidt B, Asztalos EV, Roberts RS, et al. Impact of bronchopulmonary dysplasia, brain injury and severe retinopathy on the outcome of extremely low-birth-weight infants at 18 months. results from the trial of indomethacin prophylaxis in preterms. JAMA. 2003;289(9):1124-9.

15. Sarkar S, Kaplan C, Wiswell TE, et al. Histological Chorioamnionitis and the risk of early Intraventricular hemorrhage in infants born $\leq 28$ weeks gestation. J Perinatol. 2005;25(12):749-52..

16. Hornika CP, Fort P, Clarkc RH, et al. Early and late onset sepsis in very-lowbirth-weight infants from a large group of neonatal intensive care units. Early Hum Dev. 2012;88 Suppl 2:69-74.

17. Roberts I. Neonatal thrombocytopenia: causes and management. Arch Dis Childhood Fetal Neonatal Ed. 2003;88(5):359F-364.

18. Gotsch F, Romero R, Kusanovic JP, et al. The fetal inflammatory response syndrome. Clin Obstet Gynecol. 2007;50(3):652-83.

19. Yoon BH, Romero R, Moon J, et al. Differences in the fetal Interleukin-6 response to microbial in of the amniotic cavity between term and preterm gestation [J]. J Mater Fetal Med. 2003;13(1):32-8.

20. Park CW, Moon KC, Park JS, et al. The involvement of human amnion in histologic chorioanmionitis is an indicator that a fetal and an intra-amniotic inflammatory response is more likely and severe:clinical implications. Placenta. 2009;30:56-61.

21. Gomez R, Romero R, Ghezzi F, et al. The fetal inflammatory response syndrome. Am J Obstet Gynecol. 1998;179(1):194-202.

22. Norwitz ER. Defective implantation and placentation: laying the blueprint for pregnancy complications. Reprod BioMed Online. 2006;13(4):591-9.

23. Rees $\mathrm{S}$, Inder $\mathrm{T}$. Fetal and neonatal origins of altered brain development. Early Hum Dev. 2005;81(9):753-61.

24. Kelly $R$, Holzman C, Senagore P, et al. Placental vascular pathology findings and path- ways to preterm delivery. Am J Epidemiol. 2009;170:148-58.

25. McNamara H, Hutcheon JA, Platt RW, et al. Risk factors for high and low placental weight. Paediatr Perinat Epidemiol. 2014;28:97-105.

26. Holzman C, Lin X, Senagore P, et al. Histologic chorioamnionitis and preterm delivery. Am J Epidemiol. 2007;166:786-94.

27. Pinar $\mathrm{H}$, Goldenberg RL, Koch MA, et al. Placental findings in singleton stillbirths. Obstet Gynecol. 2014;123:325-36.

28. Erdemir G, Kultursay N, Calkavur S, et al. Histological Chorioamnionitis: effects on premature delivery and neonatal prognosis. Pediatr Neonatol. 2013:54:267-74
29. Zanardo V, Vedovato S, Suppiej A, et al. Histological inflammatory responses in the placenta and early neonatal brain injury. Pediatr Dev Pathol. 2008: 11(5):350-4.

30. Vergani P, Patane L, Doria P, et al. Risk factors for neonatal Intraventricular Haemorrhage in spontaneous prematurity at 32 weeks gestation or less. Placenta. 2000;21:402-7..

31. Toti P, De Felice C. Chorioamnionitis and fetal/neonatal brain injury. Biol Neonate. 2001;79:201-4.

32. Dammann O, Leviton A. Infection remote from the brain, neonatal white matter damage, and cerebral palsy in the preterm infant. Semin Pediatr Neurol. 1998;5:190-201.

33. Wells JT, Ment LR. Prevention of intraventricular hemorrhage in preterm infants. Early Hum Dev. 1995;42:209-33.

34. Lou HC. The "lost autoregulation hypothesis" and brain lesions in the newbornFan update. Brain and Development. 1988;10:143-6.

35. Watterberg KL, Scott SM, Naeye RL. Chorioamnionitis, cortisol, and acute lung disease in very low birth weight infants. Pediatrics. 1997;99:E6.

36. Ogunyemi D, Murillo M, Jackson U, et al. The relationship between placental histopathology findings and perinatal outcome in preterm infants. J Matern Fetal Neonatal Med. 2003;13:102e9.

37. Strunk T, Doherty D, Jacques $A$, et al. Histologic chorioamnionitis is associated with reduced risk of late-onset sepsis in preterm infants. Pediatrics. 2012;129:e134e41

38. Azizia M, Lloyd J, Allen $\mathrm{M}$, et al. Immune status in very preterm neonates. Pediatrics. 2012;129:e967e74.

39. Dammann O, Brinkhaus MJ, Bartels DB, et al. Immaturity, perinatal inflammation, and retinopathy of prematurity: a multi-hit hypothesis. Early Hum Dev. 2009;85:325-9.

40. Duci M, Frigo AC, Visentin S, et al. Maternal and placental risk factors associated with the development of necrotizing enterocolitis (NEC) and its severity. J Pediatr Surg. 2019;54(10):2099-102.

41. Been JV, Degraeuwe PL, Kramer BW, et al. Antenatal steroids and neonatal outcome after chorioamnionitis: a meta-analysis. BJOG. 2011;118:113e22.

42. Tauscher MK, Berg D, Brockmann M, et al. Association of Histologic Chorioamnionitis, increased levels of cord blood cytokines, and Intracerebra hemorrhage in preterm neonates. Neonatology. 2003;83(3):166-70.

\section{Publisher's Note}

Springer Nature remains neutral with regard to jurisdictional claims in published maps and institutional affiliations.
Ready to submit your research? Choose BMC and benefit from:

- fast, convenient online submission

- thorough peer review by experienced researchers in your field

- rapid publication on acceptance

- support for research data, including large and complex data types

- gold Open Access which fosters wider collaboration and increased citations

- maximum visibility for your research: over $100 \mathrm{M}$ website views per year

At BMC, research is always in progress.

Learn more biomedcentral.com/submissions 